\title{
Comparing Optical Coherence Tomography Radial and Cube Scan Patterns for Measuring Bruch's Membrane Opening Minimum Rim Width (BMO-MRW) in Glaucoma and Healthy Eyes: Cross-sectional and Longitudinal Analysis
}

A thesis submitted to the University of Arizona College of Medicine - Phoenix in partial fulfillment of the requirements for the degree of Doctor of Medicine

Sami W. Kabbara

Class of 2018

Mentor: Akram Belghith, PhD 
Background and Significance: Spectral Domain-Optical Coherence Tomography (OCT) is one of the most widely used imaging modality in Ophthalmology. It utilizes light waves to visualize the various layers of the retina. The OCT machines offer two different scan patterns, the circular and the cube scan patters. It is important to compare these scan pattern to see if any discrepancy exist in quantifying retinal indices. One of the newer indices is the Bruch's membrane opening minimum rim width (BMO-MRW), which is the minimum distance between from the $\mathrm{BMO}$ to the inner limiting membrane (ILM). The BMO-MRW is being used in the diagnosis of glaucoma.

Hypothesis: To compare the cube and radial scan patterns of the SD-OCT for quantifying the BMO-MRW. We hypothesis that there might be some differences between the two scan patterns.

Methods Sixty healthy eyes and 189 glaucomatous eyes were included. The optic nerve head cube and radial pattern scans were acquired using Spectralis SD-OCT. BMO-MRWs were automatically delineated using the San Diego Automated Layer Segmentation Algorithm. The BMO-MRW diagnostic accuracy for glaucoma detection and rates of change derived from the two scan patterns were compared.

Results: There was a significant difference between the baseline global BMO-MRW measurements of cube and radial scans for healthy $(301.9 \pm 57.8 \mu \mathrm{m}$ and $334.7 \pm 61.8 \mu \mathrm{m}$, respectively, $\mathrm{p}=0.003)$ and glaucoma eyes $(181.2 \pm 63.0 \mu \mathrm{m}$ and $210.2 \pm 67.2 \mu \mathrm{m}$, respectively, $\mathrm{p}<0.001)$. The area under the receiver operating characteristic curve for differentiating between healthy and glaucoma eyes was 0.90 for both the radial scan based and cube scan-based BMOMRW. No significant difference in the rate of BMO-MRW change (mean follow-up years) by scan pattern was found among both healthy (cube: $-1.47 \mu \mathrm{m} /$ year, radial: $-1.53 \mu \mathrm{m} /$ year; $\mathrm{p}=0.48$ ) (1.6 years) and glaucoma eyes (cube: $-2.37 \mu \mathrm{m} /$ year, radial: $-2.28 \mu \mathrm{m} /$ year; $\mathrm{p}=0.45$ ) (2.6 years).

Conclusion: Although the cube scan-based BMO-MRW was significantly smaller than the radial scan-based BMO-MRW, we found no significant difference between the two scan patterns for detecting glaucoma, identifying BMO location and measuring the rate of BMO-MRW change. 
These results suggest that although BMO-MRW estimates are not interchangeable, both scan patterns can be used for monitoring BMO-MRW changes over time. 


Table of Contents


List of Figures and Tables

Table 1: Baseline demographics and ocular characteristics by group

Table 2: BMO location differences between cube and radial scan values

Table 3: Comparison of BMO-MRW baseline values obtained from the cube and radial scan patterns

Figure 2: Bland-Altman Plot showing agreement between radial and cube scan patterns between baseline global BMO-MRW values in glaucoma and healthy eyes

Figure 3: Bland Altman Plot showing agreement between radial and cube scan patterns baseline global BMO-MRW values in glaucoma eyes

Table 4: Pearson correlation between BMO-MRW Baseline values obtained from the cube and radial scan

Table 5: Rate of BMO-MRW change obtained from the cube and radial scans

Figure 4: Inverted histogram showing the distribution of rate of global BMO-MRW change obtained from the cube and radial scans in healthy eyes

Figure 5: Inverted histogram showing the distribution of rate of global BMO-MRW change obtained from the cube and radial scan in glaucoma eyes

Figure 6: Age, disc size and axial length-adjusted ROC curves for global BMO-MRW derived from radial and cube scan patterns, AUC, area under the ROC curb 
Introduction:

Glaucoma is a neurodegenerative disease that is characterized by accelerated retinal ganglion cell (RGC) death leading to progressive visual field loss.1 2 It is estimated that $30 \%-50 \%$ of RGCs are lost before visual field defects can be confirmed by standard visual field testing.3 4 Therefore, early detection of glaucomatous neuronal loss is critical in the diagnosis of the disease and the maintenance of vision.

Developments in spectral domain optical coherence tomography (SD-OCT) have facilitated better visualization and evaluation of microstructures that are undetectable funduscopically.57 One structure that is easily visualized by SD-OCT is the Bruch's membrane (BM). BM is a pentalaminar structure composed of the retinal pigment epithelium basement membrane, inner collagenous layer, middle elastic layer, outer collagenous layer and the choroidal endothelial cell basement membrane.8 The innermost edge of the BM, known as Bruch's membrane opening (BMO), has been shown to have clinical and diagnostic value for glaucoma.9 The BMO identifies the anatomic position of the disc margin more accurately and consistently than traditional techniques that rely on the subjective appearance of optic nerve head (ONH) surface.9 The BMO has been shown to be stable over time in both healthy and glaucomatous eyes, making it a valuable reference point for other eye structures.10

From the BMO, new indices have been introduced, such as the BMO minimum rim width (BMOMRW), which is defined as the minimum distance from the BMO to the internal limiting membrane (ILM).9 Some studies suggest that the BMO-MRW is a better predictor of visual field total deviation and visual sensitivity threshold than retinal nerve fibre layer (RNFL) thickness in patients with glaucoma.11 12 In contrast, other studies have found that the diagnostic accuracy of BMO-MRW is similar to RNFL thickness for differentiating between healthy and glaucoma eyes.13

Because of the potential clinical importance of BMO and BMO-MRW indices, it is important to characterize how different scan patterns influence the measurements of these two indices. The cube and radial scan patterns are the most widely used in SD-OCT. The cube scan comprised parallel and equidistant B-scans, while the radial scan comprised 48 B-scans at constant angular 
intervals centered on the ONH. The Spectralis OCT (HRA+ OCT) has both scan patterns available for imaging the ONH, while the Cirrus OCT focuses only on the cube scan. Previous studies have compared various image patterns; however, none have studied the differences between the cube and radial B-scan patterns in identifying the BM parameters.14-17 Utilizing the Spectralis SD-OCT, we examined differences between the two scan patterns for measuring the BMO location, the BMO-MRW and the rate of BMO-MRW change over time. We hypothesis that we will find some differences in calculating the BMO-MRW between the cube and the circular scan. 
Methods

Participants

The participants of this study were recruited from the Diagnostic Innovations in Glaucoma Study (DIGS) at the University of California, San Diego, which was designed to examine the visual function and optic nerve structure in patients with glaucoma. Methodological details about DIGS have been outlined previously.18 Methods of this study abide by the Declaration of Helsinki and the Health Insurance Portability and Accountability Act. The protocol was approved by the University of California San Diego Human Subjects Committee and the University of California, San Diego Institutional Review Board. In short, inclusion criteria for all subjects (healthy and glaucoma) were (1) 20/40 visual acuity or better, (2) spherical refraction of less than \pm 5.0 dioptre and (3) cylinder correction of less than \pm 3 dioptre.

For healthy participants, further inclusion criteria include (1) an intraocular pressure (IOP) $<22$ $\mathrm{mm} \mathrm{Hg}$ with no previous history of elevated IOP and (2) a minimum of two normal visual field tests using the standard automated perimetry (SAP) with a pattern standard deviation (PSD) within 95\% confidence limits and a glaucoma hemifield test (GHT) within normal limits.

For participants with glaucoma, further inclusion criteria include (1) open angles on gonioscopy, and (2) at least two consecutive abnormal SAP tests with either PSD or GHT result outside 99\% normal limits. Exclusion criteria include eyes with any optic neuropathy other than glaucoma.

This study included 249 eyes of 130 subjects (60 eyes of 30 healthy subjects and 189 eyes of 100 glaucoma subjects). Standard assessments of simultaneous stereophotographs were also used to identify optic disc changes in glaucoma subjects. If at least one eye exhibited glaucomatous visual field loss, the subject would be considered in the glaucoma group. Furthermore, in order to be considered in the longitudinal data analysis, subjects were required to have a minimum total of three SAP and three OCT tests and 1.2 years of follow-up.

Imaging

ONH scans were acquired using Spectralis SD-OCT (Heidelberg Engineering, Heidelberg, Germany). Images were analysed with the Heidelberg V.6.0.9.0 software. The acquisition rate 
of the device is $40000 \mathrm{~A}$-scans per second at an axial resolution of $3.9 \mu \mathrm{m}$. Two ONH scan types were acquired, the cube scan and the radial scan pattern. The $4.5 \times 4.5 \mathrm{~mm}$ cube scan comprised $30^{\circ}$ wide, $73 \mathrm{~B}$-scans with $768 \mathrm{~A}$-scans per B-scan. The resolution of the $\mathrm{ONH}$ cube scan is $73 \times 768 \times 496$ pixels. The B-scans are separated by $0.06 \mathrm{~mm}$, while the A-scans are separated by $0.006 \mathrm{~mm}$. The radial scan comprised $30^{\circ}$ wide, $48 \mathrm{~B}$-scans with $1024 \mathrm{~A}$-scans per B-scan. The resolution of the $\mathrm{ONH}$ radial scan is $48 \times 1096 \times 496$ pixels.

The Spectralis OCT incorporates a real-time eye tracking system to reduce eye motion artefact and reduce variability in follow-up scans. For each scan pattern (ie, cube scan and radial scan), the follow-up image is registered automatically by instrument software. Quality assessment of OCT scans was completed by the Imaging Data Evaluation and Analysis Centre examiners, who were masked to the subject's diagnosis and other test results. Good quality scans required good focus and a signal-to noise ratio $\geq 15 \mathrm{~dB}$.

Image segmentation

To calculate the MRW in both cube and radial scans, raw Spectralis ONH cube and radial scans were exported to a numerical computing language (MATLAB; MathWorks, Natick, Massachusetts, USA). The automated segmentation algorithm, San Diego Automated Layer Segmentation Algorithm (SALSA), was used to automatically segment the BMO-MRW, the shortest distance between BM opening and the ILM. Details of the SALSA have been described previously.10 1920 The positions of the BMO points were identified automatically through SALSA.

Image alignment

To compare the BMO detection results derived from the radial and the cube scans, an $x-y$ axes registration is performed by a custom free field registration software using the blood vessels in the en face image, which has the benefit of not introducing bias into the estimate of the variation in BMO location because the registration is performed on the en face image and not 
on each individual B-scan. Moreover, the $x-y$ registration is necessary to ensure that we are comparing the same region over time.

Statistical analysis

Descriptive statistics were used to compare demographic characteristics of healthy and glaucoma eyes. t-tests were used to compare continuous variables and $\mathrm{X} 2$ tests were used to compare categorical variables.

For the SALSA BMO identification method, the difference of the BMO location derived from the cube and radial scan was calculated. The analysis was performed with commercial statistical software package (SAS System for Windows, Version 8.2, SAS Institute, Cary, North Carolina, USA) using the minimum norm quadratic unbiased estimation method. Differences in the intercept and slope between healthy and progressing glaucoma eyes were modelled as fixed effects, and the individual distribution of intercept and slope between subjects was modelled as random effects. The model was adjusted for age and the correlation between eyes was accounted for in the model. BMO-MRW thicknesses between the two scan patterns were compared using the paired t-test. The relationship between two scan patterns was assessed using Pearson correlation coefficients. 
Results

Sixty eyes of 30 healthy subjects and 189 eyes of 100 glaucoma subjects were included. The glaucoma group consisted of 144 early glaucoma eyes (mean deviation (MD)>-6 dB), 26 moderate glaucoma eyes $(-12<\mathrm{MD}-\leq 6)$ and 19 advanced glaucoma eyes (MD $\leq-12 \mathrm{~dB})$. The demographic and baseline ocular characteristics of each study group are represented in table 1. The mean $( \pm S D)$ age of patients with glaucoma ( $71 \pm 11$ years; range 34-92 years) was significantly older than healthy subjects ( $48 \pm 12$ years; range $24-68$ years, $p<0.001$ ). Compared with the healthy group, the glaucoma group had a smaller proportion of women (77\% vs $49 \%$, respectively, $p=0.003$ ), individuals of African descent ( $30 \%$ vs $18 \%$, respectively, $p=0.001$ ) and longer duration of follow-up (mean 1.6 years (range 1.2-1.8) vs 2.6 years (range 2.1-3.4) respectively, $p<0.001)$. No statistically significant differences were found between the glaucoma and healthy eyes with regard to mean IOP during follow-up $(\mathrm{mm} \mathrm{Hg})$ and disc area $(\mathrm{mm} 2)$ $(p=0.381$ and $p=0.065$, respectively). 


\begin{tabular}{|c|c|c|c|}
\hline By Subject & $\begin{array}{l}\text { Healthy } \\
\mathrm{N}=30\end{array}$ & $\begin{array}{c}\text { Glaucoma } \\
\mathrm{N}=100\end{array}$ & P Value \\
\hline Mean age at baseline (years) & $48 \pm 12$ & $71 \pm 11$ & $<0.001$ \\
\hline Gender, Female (\%) & $23(77 \%)$ & $49(49 \%)$ & 0.003 \\
\hline Race: European Descent & $21(70 \%)$ & $81(81 \%)$ & \multirow{3}{*}{$<0.001$} \\
\hline African Descent & $9(30 \%)$ & $18(18 \%)$ & \\
\hline Other & & $1(1 \%)$ & \\
\hline By Eye & $\mathrm{N}=60$ & $\mathrm{~N}=189$ & \\
\hline $\begin{array}{l}\text { Mean IOP during follow-up } \\
(\mathrm{mmHg})\end{array}$ & $14.8 \pm 2.8$ & $15.3 \pm 4.4$ & 0.381 \\
\hline Disc area $\left(\mathrm{mm}^{2}\right)$ & $1.93 \pm 0.45$ & $2.07 \pm 0.53$ & 0.065 \\
\hline Mean Spherical Equivalent (D) & $-0.41 \pm 1.26$ & $-1.22 \pm 1.97$ & 0.004 \\
\hline Mean Axial Length (mm) & $23.7 \pm 0.8$ & $24.2 \pm 1.14$ & 0.002 \\
\hline Baseline MD (dB) & $-0.05 \pm 1.26$ & $-3.7 \pm 5.3$ & $<0.001$ \\
\hline Mean No. of Spectralis visits, (IQR) & $5(3-6)$ & $7(5-9)$ & $<0.001$ \\
\hline Mean Follow up (years) (IQR) & $1.6(1.2-1.8)$ & $2.6(2.1-3.4)$ & $<0.001$ \\
\hline
\end{tabular}

*values are presented as mean \pm SD unless otherwise indicated 
The difference in global and sectoral BMO location measured in voxels between the cube and radial scans in both glaucoma and normal eyes was not significant ( $p=0.48$ for global) (table 2). Specifically, in glaucoma eyes, the mean global BMO location difference between cube and radial scans was 2.25 voxels ( $S D \pm 2.01$ ). The inferior sector had the largest difference in $\mathrm{BMO}$ location between cube and radial scans ( $2.50 \pm 2.53$ voxels), while the temporal sector had the smallest difference (1.70 \pm 1.27 voxels). In healthy subjects, the mean global BMO location difference between the cube and radial scan was $2.14 \pm 1.33$ voxels. The largest difference between scan types was found in the inferior sector ( $2.36 \pm 1.72$ voxels), and the smallest difference was in the nasal sector (1.82 \pm 1.40 voxels). 


\begin{tabular}{|c|c|c|c|}
\hline \multicolumn{5}{|c|}{ Table 2. BMO Location Difference Between Cube and Radial Scan } \\
Voxels, mean \pm SD
\end{tabular}


A Bland-Altman plot was constructed to compare the $\mathrm{x}$-axis locations of two BMO points derived from the radial and cube scans. For each of the 48 radial scans, two BMO locations were identified resulting in 96 points for comparison with the cube scan, which was registered in the $y$-axis and z-axis to the radial scan. The Bland-Altman plot indicates that there is no bias detected among the differences in BMO location measurements between the radial and cube scans (figure 1). The $95 \%$ limits of agreement were -5.5 to 5.5 voxels. 


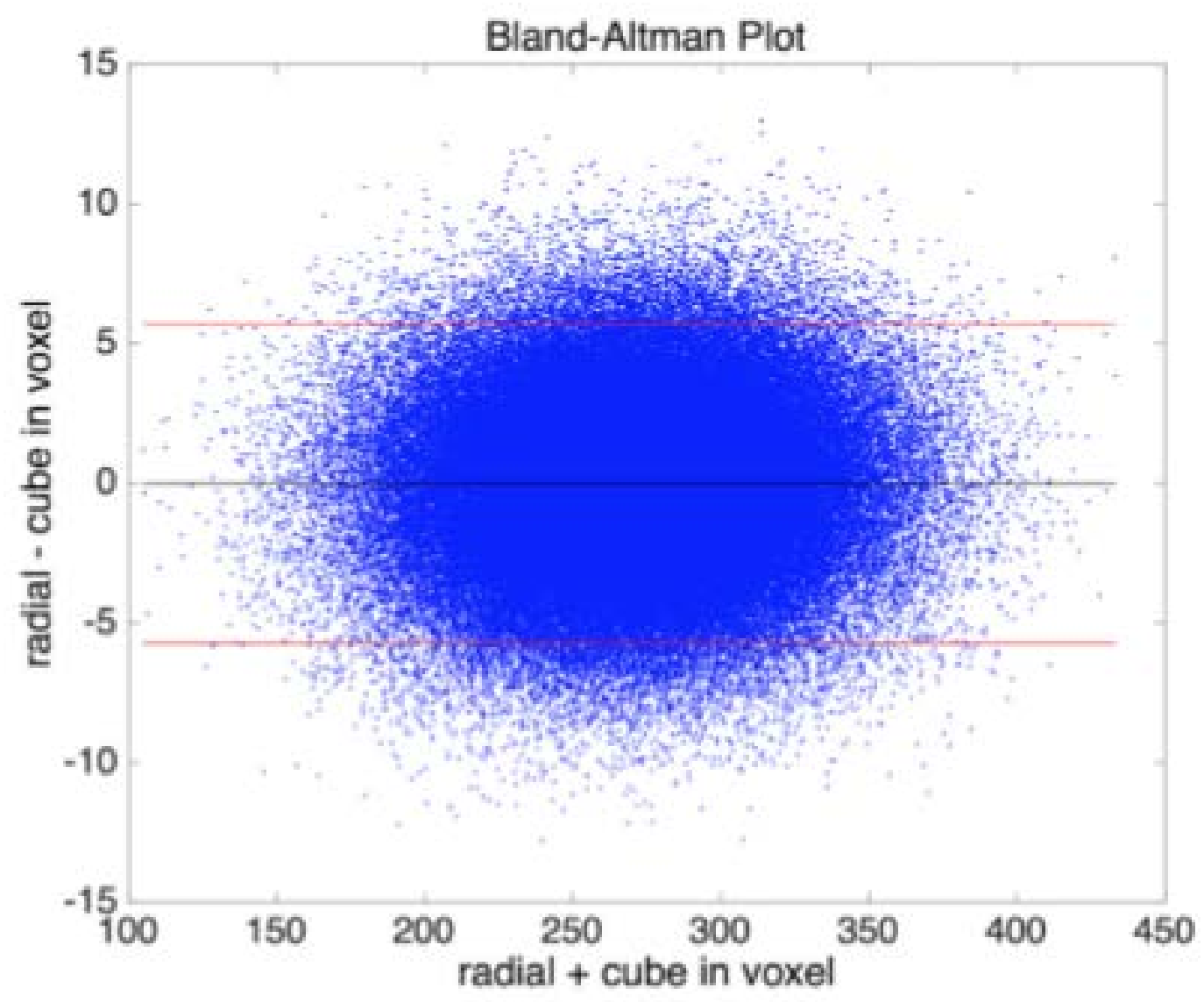

Figure 1 Bland-Altman plot shows agreement between the $x$-axis coordinates of the BMO points derived from radial and cube scan patterns. The $y$-axis and $z$-axis were not included as both cube and radial scans were aligned. No fixed bias is observed. 
Baseline global and sectorial BMO-MRW (in $\mu \mathrm{m}$ ) measurements obtained from radial and cube scans are presented in table 3 . We found a significant difference $(p=0.003)$ between the baseline global and sectors BMO-MRW measurements obtained by the two scan patterns; the radial scan consistently yielded a longer BMO-MRW than the cube scan. 


\begin{tabular}{|c|c|c|c|c|c|c|}
\hline \multicolumn{4}{|c|}{ Healthy $N=60$ eyes } & \multicolumn{3}{|c|}{ Glaucoma $\mathrm{N}=189$ eyes } \\
\hline & Cube & Radial & $\begin{array}{c}\text { P-Valu } \\
\mathrm{e}\end{array}$ & Cube & Radial & P-Value \\
\hline Global & $301.8 \pm 57.8$ & $334.6 \pm 61.7$ & 0.003 & $181.2 \pm 62.9$ & $210.1 \pm 67.1$ & $<0.001$ \\
\hline Superior & $318.1 \pm 71.4$ & $348.0 \pm 67.3$ & 0.027 & $176.9 \pm 76.1$ & $210.6 \pm 80.7$ & $<0.001$ \\
\hline Inferior & $339.6 \pm 70.1$ & $371.2 \pm 77.5$ & 0.032 & $196.5 \pm 73.4$ & $211.8 \pm 79.7$ & 0.034 \\
\hline Temporal & $216.0 \pm 50.1$ & $236.4 \pm 52.5$ & 0.037 & $143.7 \pm 52.5$ & $162.1 \pm 54.7$ & $<0.001$ \\
\hline Nasal & $336.5 \pm 66.9$ & $365.5 \pm 74.6$ & 0.029 & $209.3 \pm 82.8$ & $242.4 \pm 86.7$ & $<0.001$ \\
\hline
\end{tabular}


Bland-Altman plots were used to check for bias in between the two scan patterns when measuring the global BMO-MRW in both healthy and glaucoma groups (figures 2 and 3). We found a positive correlation between the cube and radial scans baseline BMO-MRW values; the correlation coefficient was $r=0.88$ and $r=0.90$ for the global BMO-MRW measurements in healthy and glaucoma eyes, respectively ( $p$ value $<0.0001$ ) (table 4 ). In both healthy and glaucoma eyes, the correlation was weakest for the BMO-MRW in the temporal sectors ( $r=0.80$ and $r=0.85$, respectively, $p$ value $<0.001$ ). 


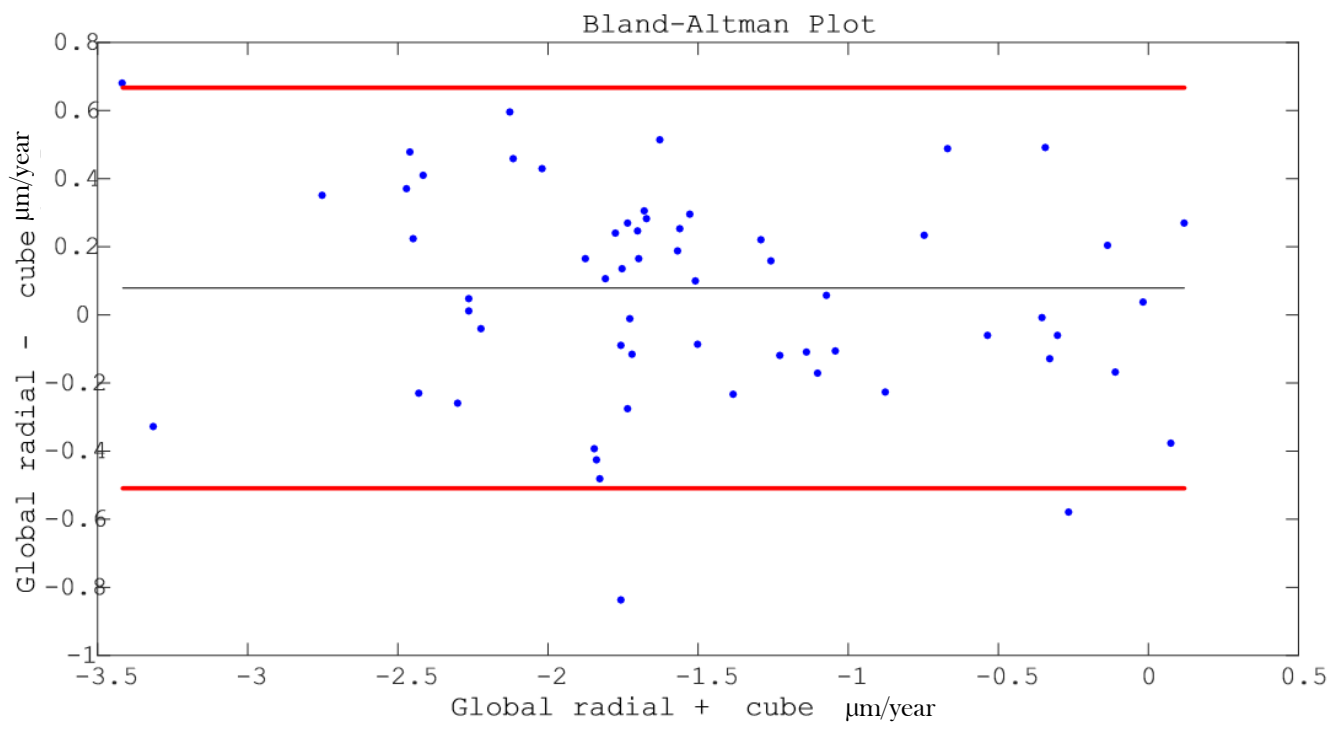

Figure 2 Bland-Altman plot shows agreement between the radial and cube scan patterns baseline global BMO-MRW values in glaucoma and healthy eyes. No fixed bias is observed. BMO-MRW, Bruch's membrane opening minimum rim width. 


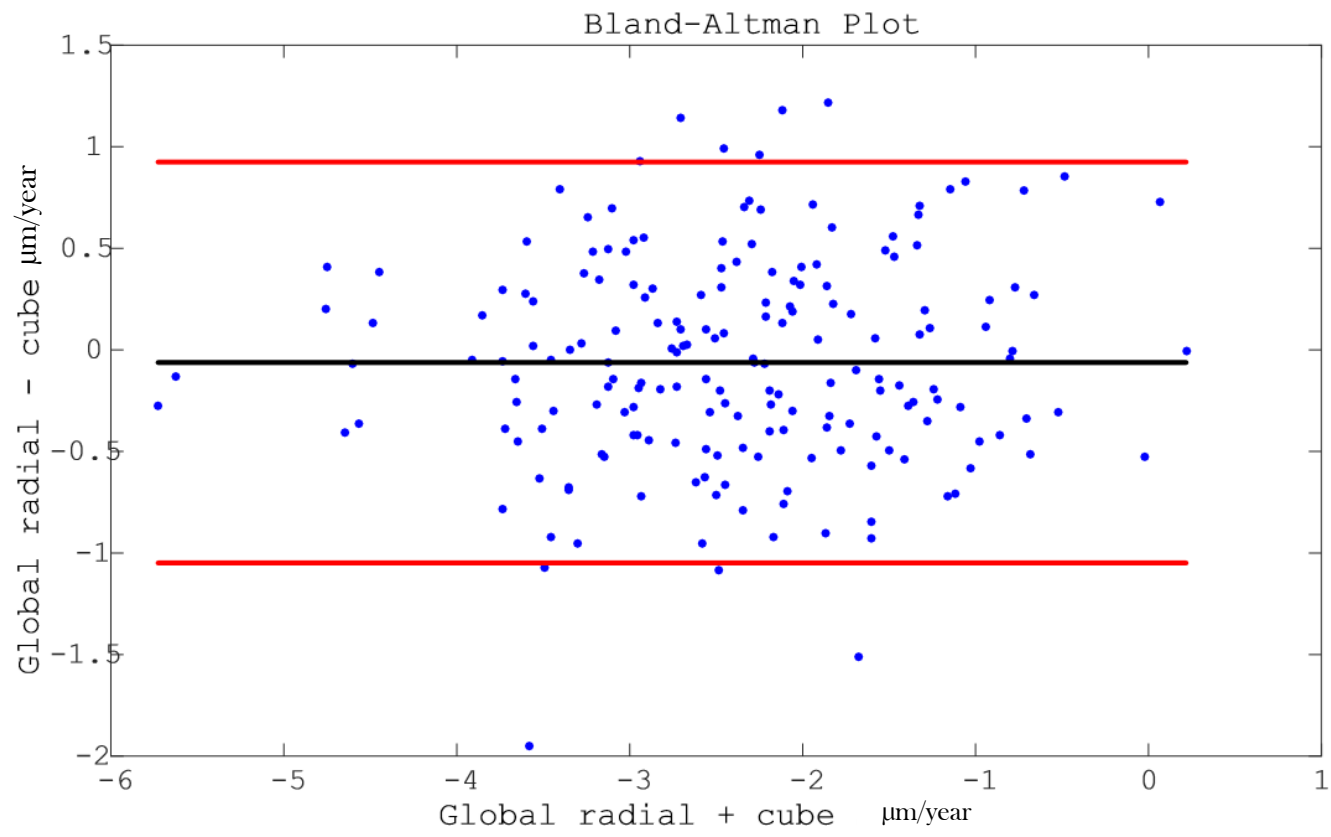

Figure 3 Bland-Altman plot shows agreement between the radial and cube scan patterns baseline global BMO-MRW values in glaucoma eyes. No fixed bias is observed. BMO-MRW, Bruch's membrane opening minimum rim width. 


\begin{tabular}{|c|c|c|c|c|}
\hline \multicolumn{5}{|c|}{$\begin{array}{l}\text { Table 4. Pearson correlation between BMO-MRW Baseline values obtained from the Cube and } \\
\text { Radial Scan }\end{array}$} \\
\hline \multicolumn{3}{|c|}{ Healthy eyes } & \multicolumn{2}{|c|}{ Glaucoma eyes } \\
\hline Sector & Correlation & p-Value & Correlation & P-value \\
\hline Global & 0.88 & $<0.001$ & 0.90 & $<0.001$ \\
\hline Superior & 0.83 & $<0.001$ & 0.88 & $<0.001$ \\
\hline Inferior & 0.90 & $<0.001$ & 0.86 & $<0.001$ \\
\hline Temporal & 0.80 & $<0.001$ & 0.85 & $<0.001$ \\
\hline Nasal & 0.88 & $<0.001$ & 0.89 & $<0.001$ \\
\hline
\end{tabular}


The rate of BMO-MRW change measured in $\mu \mathrm{m}$ per year obtained from the cube and radial scans is shown in table 5 . There was no statistical difference between the two scan patterns in the global and sectoral rates of BMO-MRW change in both healthy and glaucoma eyes. p-values ranged from 0.24 to 0.57 . The glaucoma eyes showed a significantly steeper decline in BMOMRW global rate of BMO-MRW change in cube scan $(-2.37 \mu \mathrm{m} /$ year) and radial scan $(-2.28$ $\mu \mathrm{m} /$ year) than the healthy group (global rate of BMO-MRW change in cube scan: -1.47 $\mu \mathrm{m} /$ year; radial scan: $-1.53 \mu \mathrm{m} /$ year) $(\mathrm{p}=0.45$ and $\mathrm{p}=0.48$, respectively). 


\begin{tabular}{|c|c|c|c|}
\hline \multicolumn{5}{|c|}{ Table 5. Rate of BMO-MRW Change obtained from the Cube and Radial Scans } \\
mean [95\% Cl] $(p$ value $)$
\end{tabular}

* $\mathrm{p}$-value =difference between rate of changes measured by Cube and Radial scans. 
The distribution of BMO-MRW rates of change obtained from cube and radial scans in healthy and glaucoma eyes is presented in figures 4 and 5 . In both groups, the majority of eyes fell between -1 and $-3 \mu \mathrm{m} /$ year. The rates of change of BMO-MRW exhibited similar distribution between the cube and radial scans in both healthy and glaucoma eyes. 


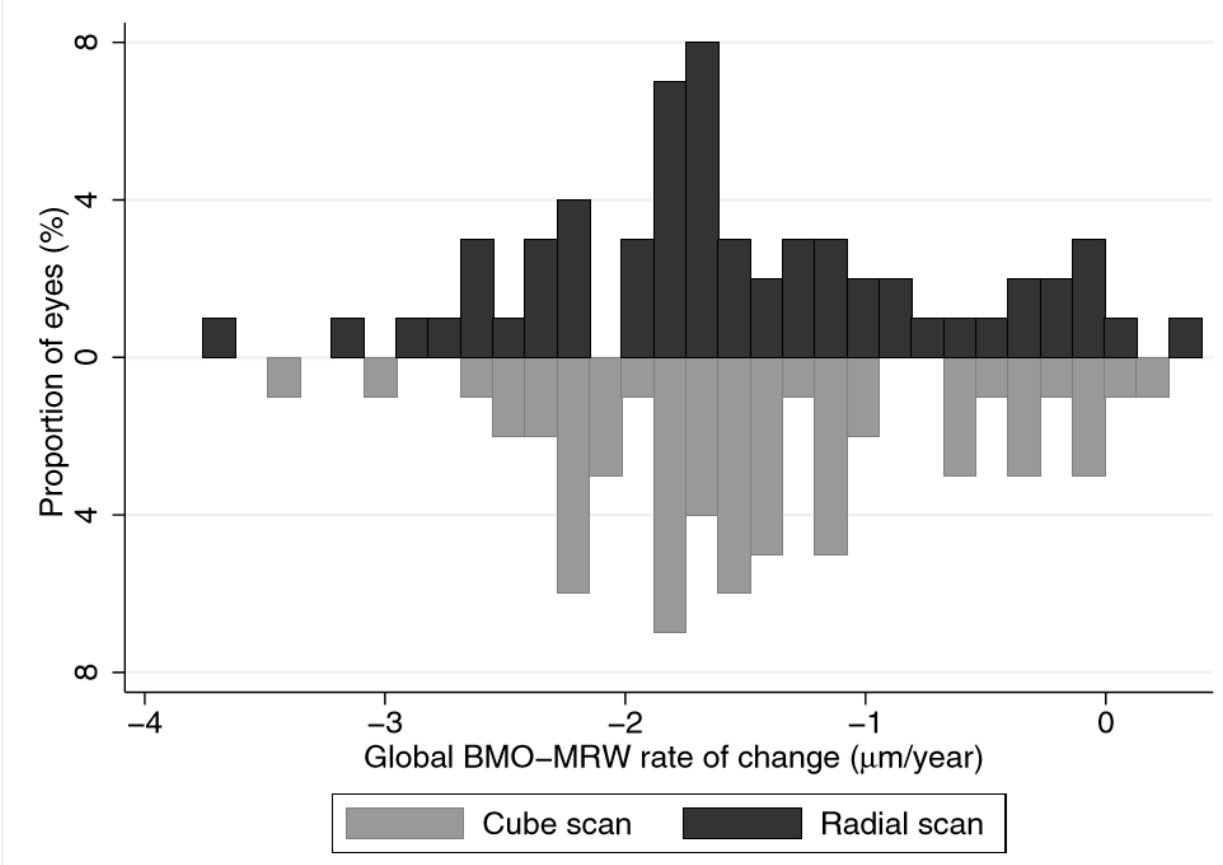

Figure 4 Inverted histogram showing the distribution of rate of global BMO-MRW change obtained from the cube and radial scans in healthy eyes. BMO-MRW, Bruch's membrane opening minimum rim width. 


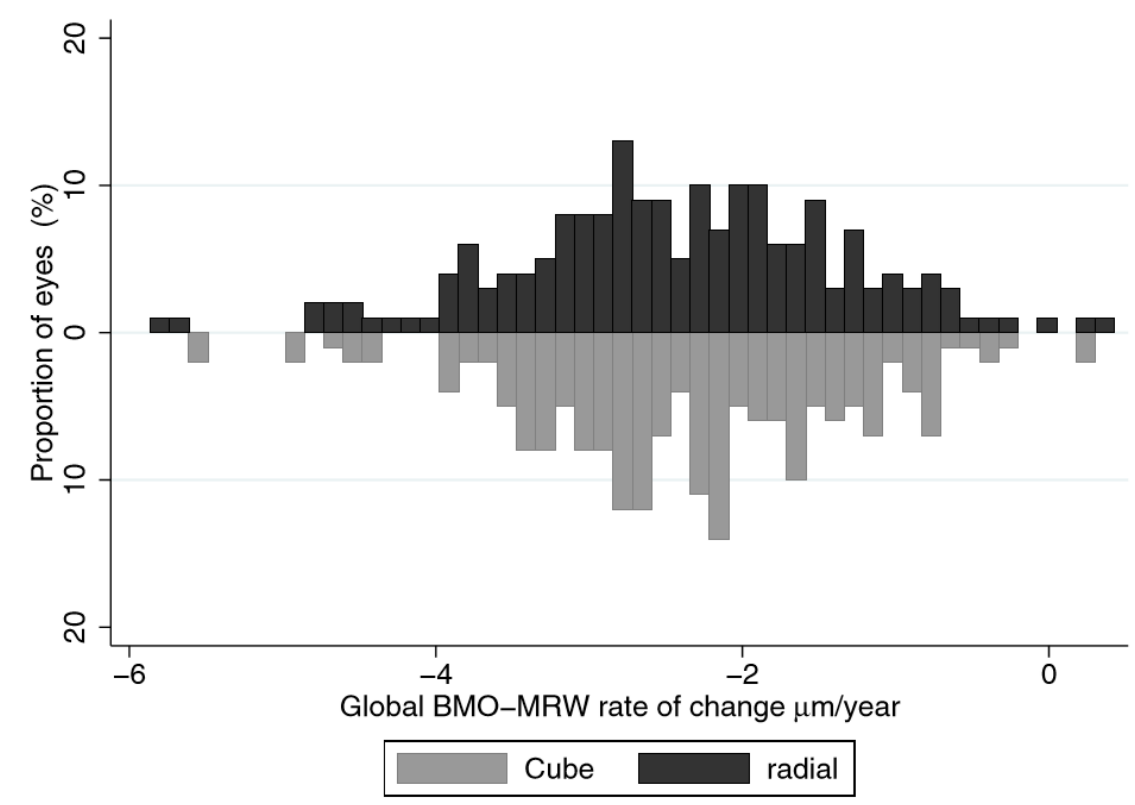

Figure 5 Inverted histogram showing the distribution of rate of global BMO-MRW change obtained from the cube and radial scans in glaucoma eyes. BMO-MRW, Bruch's membrane opening minimum rim width. 
The AUC for differentiating between healthy and glaucoma eyes using global BMO-MRW was 0.90 for both the radial scan based $(95 \% \mathrm{Cl} 0.83$ to 0.96$)$ and cube scan-based $(95 \% \mathrm{Cl} 0.83$ to 0.97) BMO-MRW, after adjusting for disc size, age and axial length (figure 6). 


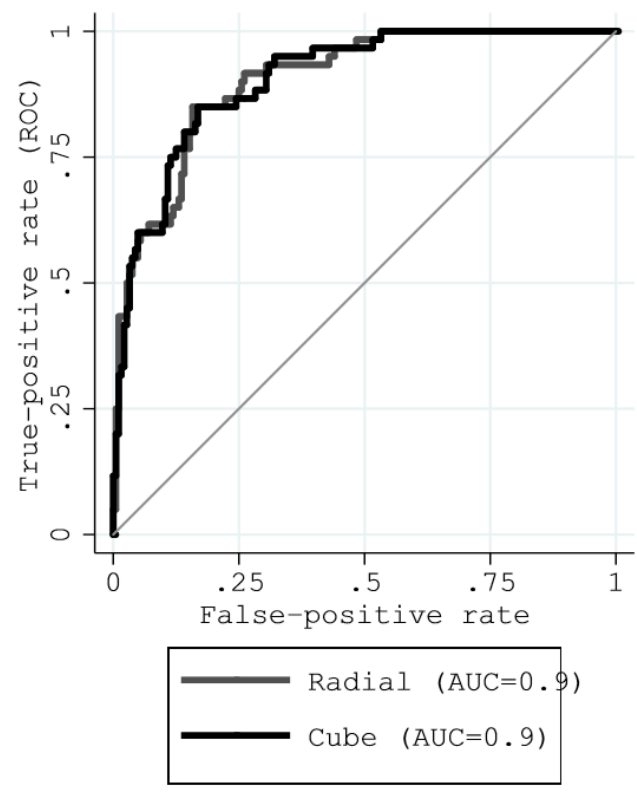

Figure 6 Age, disc size and axial length-adjusted ROC curves for global BMO-MRW derived from radial and cube scan patterns. AUC, area under the ROC curve; BMO-MRW, Bruch's membrane opening minimum rim width; ROC, receiver operating characteristic. 


\section{Discussion}

In this longitudinal study, we examined the differences between cube and radial B-scan patterns in measuring the baseline BMO location and BMO-MRW, and the rate of BMO-MRW change. We found no significant difference in BMO location between the two scan patterns, with a small average difference of two voxels between the two scan patterns. However, we found notable and significant differences in the baseline BMO-MRW between the two scan patterns $(p=0.003$ and $p<0.001$ for global measurement of healthy and glaucoma eyes, respectively). Despite differences in baseline BMO-MRW values, we found no significant differences in the rate of BMO-MRW change obtained by radial and cube scan patterns $(p=0.48$ for global rate of BMO-MRW change in healthy group and $\mathrm{p}=0.45$ for global rate of BMO-MRW change in glaucoma eyes). These findings suggest radial and cube scans will produce similar results when measuring BMO-MRW progression if the data from the two scan patterns are analyzed separately.

While this study compares radial and cube scan patterns in relation to BMO-MRW, several other studies have compared the effectiveness of radial and cube scans in measuring parameters other than BMO-MRW. In one such study, Schneider et al22 studied the differences between several scan patterns and densities when evaluating macular holes. They looked at the three scan patterns (61-line cube volume, 6 -line and 24-line radial scan) and found the 24-line radial scan to provide better detection of small full-thickness macular holes in comparison to the other two scan patterns.22 Mayama et al15 looked at the difference in performance between circular scan, cube scan and annular scan patterns in measuring the peripapillary RNFL thickness. The cube scan was shown to have greater sensitivity than the circular and the annular method in detecting early-stage glaucoma. Our study differs in that we used the cube and radial image patterns to look at a different optic structure, the $\mathrm{BM}$, near the $\mathrm{ONH}$. Moreover, our images were automatically segmented and analysed using SALSA, which facilitates a more objective assessment, as opposed to the manual segmentation carried out by Schneider et al.22 
Since RGC axons that comprise the RNFL cannot penetrate the underlying BM, all axons are confined to the BMO-MRW space. This phenomenon offers a new potential index in assessing the progression of glaucoma, the BMO-MRW. Gardiner et al23 have shown that SD-OCTderived measurements of RNFL thickness correlated well with BMO-MRW ( $r=0.680)$, while Chauhan et al24 found BMO-MRW to be a better diagnostic marker in the detection of glaucoma than RNFL thickness. Moreover, Tun et al25 in a cross-sectional study of healthy ethnic Chinese subjects found that rim area $(\beta=194.31, p<0.001)$ was more strongly associated with BMO-MRW than RNFL thickness $(\beta=0.5, p=0.04)$. As expected from the neurodegenerative nature of glaucoma, we found that BMO-MRW rate of decline measured by both the cube and radial B-scans was higher in glaucoma than normal group due to more RGC loss and RNFL thinning (cube: $-1.47 \mu \mathrm{m} /$ year, radial: $-1.53 \mu \mathrm{m} /$ year ( $\mathrm{p}$ value: 0.48 ) for healthy; cube: -2.37 $\mu \mathrm{m} /$ year, radial: $-2.37 \mu \mathrm{m} /$ year ( $p$ value: 0.45 ) for glaucoma).

In addition to the BMO-MRW, the optic rim and the cup-to disc ratio can also be derived from the BMO. Strouthidis et al26 have shown that depending on the tissue architecture, the BMO constitutes the boundaries for disc margin. Since we found that the average difference between the BMO locations in the radial and cube scans was relatively small (average difference of two voxels), both scan patterns can be equally used to determine the disc margin. However, it is important to note that $\mathrm{BMO}$ does not always correlate to what clinicians perceive as the disc margin. In myopic eyes with disc tilt, BMO might not necessarily match with the clinical disc margin. 26

There are several limitations in this study. First, subjects in this study were exclusively imaged with Spectralis SD-OCT (Heidelberg Engineering), which might limit the generalisability of our findings to other OCT instruments. Second, the images were captured by the Spectralis SD-OCT on the high-resolution setting, which has higher resolution and a relatively slow acquisition speed compared with the high-speed setting. The highspeed setting on the Spectralis may be more practical in the clinical environment, as the acquisition time is faster and requires less focus by the patient. Additional studies need to be completed to verify if our results are consistent with the high-speed setting. Third, the cube scans comprised B-scans that are separated by $0.6 \mathrm{~mm}$, while the A-scans are separated by $0.006 \mathrm{~mm}$. Shorter distances between 
B-scans will lead to a more precise quantification of the BMO-MRW. Finally, a longer patient follow-up duration would allow for better assessment of whether the rate of BMO-MRW change differs by scan pattern.

Moreover, our results showed a similar ability of the BMO-MRW derived from radial or cube scans to differentiate between healthy and glaucoma eyes. One limitation of this study is that the healthy and glaucoma groups are not age-matched, gender-matched and ethnicitymatched. For this reason, the AUC was adjusted for age, disc size and axial length, as the ethnicity and the gender were not significant in the model. 
Future Directions

In the future, we aim to study the role of other OCT scan patterns in the quantification of BMOMRW indices. We also aim to repeat our study when we have enough patients with longer follow-up duration. 


\section{Conclusions}

In summary, we demonstrated that although the cube scan based BMO-MRW was significantly smaller than the radial scan based BMO-MRW, we found no significant difference between the two scan patterns for detecting glaucoma, identifying BMO locations and measuring the rate of BMO-MRW change. These results suggest that although BMO-MRW estimates are not interchangeable, both scan patterns can be used for monitoring BMO-MRW changes over time. 


\section{References}

1 Quigley HA, Broman AT. The number of people with Glaucoma worldwide in 2010 and 2020. Br J Ophthalmol 2006;90:262-7.

2 Weinreb RN, Aung T, Medeiros FA. The pathophysiology and treatment of Glaucoma: a review. JAMA 2014;311:1901-11.

3 Quigley HA, Addicks EM, Green WR, et al. Optic nerve damage in human Glaucoma. II. the site of injury and susceptibility to damage. Arch Ophthalmol 1981;99:635-49.

4 Harwerth RS, Wheat JL, Fredette MJ, et al. Linking structure and function in Glaucoma. Prog Retin Eye Res 2010;29:249-71.

5 Srinivasan VJ, Adler DC, Chen Y, et al. Ultrahigh-speed optical coherence tomography for three-dimensional and en face imaging of the retina and optic nerve head. Invest Ophthalmol Vis Sci 2008;49:5103-10.

6 van Velthoven ME, Faber DJ, Verbraak FD, et al. Recent developments in optical coherence tomography for imaging the retina. Prog Retin Eye Res 2007;26:57-77.

7 Wojtkowski M, Srinivasan V, Fujimoto JG, et al. Three-dimensional retinal imaging with highspeed ultrahigh-resolution optical coherence tomography. Ophthalmology 2005;112:1734-46.

8 Hogan MJ, Alvarado J. Studies on the human macula. IV. aging changes in Bruch's membrane. Arch Ophthalmol 1967;77:410-20.

9 Reis AS, Sharpe GP, Yang H, et al. Optic disc margin anatomy in patients with Glaucoma and normal controls with spectral domain optical coherence tomography. Ophthalmology 2012;119:738-47.

10 Belghith A, Bowd C, Medeiros FA, et al. Does the Location of Bruch's Membrane Opening Change Over Time? Longitudinal Analysis Using San Diego Automated Layer Segmentation Algorithm (SALSA). Invest Ophthalmol Vis Sci 2016;57:675-82. 
11 Mizumoto K, Gosho M, Zako M. Correlation between optic nerve head structural parameters and glaucomatous visual field indices. Clin Ophthalmol 2014;8:1203-8.

12 Pollet-Villard F, Chiquet C, Romanet J-P, et al. Aptel F: structure-function relationships with spectral-domain optical coherence tomography retinal nerve fiber layer and optic nerve head measurements structure-function relationships with SD-OCT. Invest Ophthalmol Vis Sci 2014;55:2953-62.

13 Gmeiner JMD, Schrems WA, Mardin CY, et al. Schrems-Hoesl LM: comparison of Bruch's Membrane Opening Minimum Rim Width and Peripapillary Retinal Nerve

Fiber Layer Thickness in Early Glaucoma AssessmentBMO-MRW and RNFLT in Early Glaucoma Assessment. Invest Ophthalmol Vis Sci 2016;57.In Press.

14 Adam MK, Rayess N, Rahimy E, et al. Radial versus raster spectral-domain optical coherence tomography scan patterns for detection of macular fluid in neovascular age-related macular degeneration. Br J Ophthalmol 2016;100:491-4.

15 Mayama C, Saito H, Hirasawa H, et al. Circle- and grid-wise analyses of peripapillary nerve fiber layers by spectral domain optical coherence tomography in early-stage Glaucoma. Invest Ophthalmol Vis Sci 2013;54:4519-26.

16 Rahimy E, Rayess N, Maguire Jl, et al. Radial versus raster spectral-domain optical coherence tomography scan patterns for detection of macular pathology. Am J Ophthalmol 2014;158:34553.

17 Shin JW, Uhm KB, Lee WJ, et al. Diagnostic ability of retinal nerve fiber layer maps to detect localized retinal nerve fiber layer defects. Eye 2013;27:1022-31.

18 Sample PA, Girkin CA, Zangwill LM, et al. The african descent and Glaucoma evaluation study (ADAGES): design and baseline data. Arch Ophthalmol 2009;127:1136-45. 
19 Belghith A, Bowd C, Weinreb RN. A hierarchical framework for estimating neuroretinal rim area using 3D spectral domain optical coherence tomography (SD-OCT) optic nerve head (ONH) images of healthy and Glaucoma eyes. . Zangwill LM: Conference proceedings: IEEE Engineering in Medicine and Biology Society Annual Conference, 2014:2014. 3869-72.

20 Belghith A, Bowd C, Medeiros FA, et al. Zangwill LM: Automated Segmentation of Anterior Lamina Cribrosa surface: how the Lamina Cribrosa responds to intraocular pressure Change in Glaucoma eyes. New York: IEEE International Symposium on Biomedical Imaging ISBI, 2015:222-5.

21 Bland JM, Altman DG. Statistical methods for assessing agreement between two methods of clinical measurement. Lancet 1986;1:307-10.

22 Schneider EW, Todorich B, Kelly MP, et al. Effect of optical coherence tomography scan pattern and density on the detection of full-thickness macular holes. Am J Ophthalmol 2014;157:978-84.

23 Gardiner SK, Ren R, Yang H, et al. A method to estimate the amount of neuroretinal rim tissue in glaucoma: comparison with current methods for measuring rim area. Am J Ophthalmol 2014;157:540-9.

24 Chauhan BC, O'Leary N, Almobarak FA, et al. Enhanced detection of open-angle glaucoma with an anatomically accurate optical coherence tomography-derived neuroretinal rim parameter. Ophthalmology 2013;120:535-43.

25 Tun TA, Sun $\mathrm{CH}$, Baskaran M, et al. Determinants of optical coherence tomographyderived minimum neuroretinal rim width in a normal Chinese population. Invest Ophthalmol Vis Sci 2015;56:3337-44.

26 Strouthidis NG, Yang H, Reynaud JF, et al. Comparison of clinical and spectral domain optical coherence tomography optic disc margin anatomy. Invest Ophthalmol Vis Sci 2009;50:4709-18. 